\title{
Mean ST Segment Elevation
}

National Cancer Institute

\section{Source}

National Cancer Institute. Mean ST Segment Elevation. NCI Thesaurus. Code C62158.

The average elevation of the ST segment, obtained from a set of measurements of the elevation of the ST segment. 